\title{
Toxoplasmosis en Obstetricia
}

\author{
Dres.: Alberto Duarte-Contreras*, Clara Inés Duarte Barreto**, \\ Gladys Duarte de Ruiz***
}

La Toxoplasmosis es una zoonosis producida por un esporozoario del grupo de los coccídeos, el Toxoplasma Gondii que se transmite al hombre. Se encuentra ampliamente difundida por el mundo. Se le considera como un serio problema biológico y social. Cuando una mujer embarazada adquiere la infección, el toxoplasma puede pasar al feto y afectarlo severamente inhibiéndolo para su comportamiento posterior en el desempeño productivo social y material. En los últimos años ha ocupado con el cáncer y el sindrome de inmunodeficiencia adquirida los primeros planos en la literatura médica.

La embarazada transmite la toxoplasmosis al feto únicamente cuando ha adquirido la infección durante la gestación que cursa. Una infección toxoplásmica anterior al embarazo actual en nada perjudica al producto, al que defienden los anticuerpos maternos.

\section{TRANSMISION DE LA TOXOPLASMOSIS}

La embarazada se infecta:

1. Por contacto directo cuando teniendo soluciones de continuidad en sus manos manipula carnes crudas recientemente infectadas.

2. Por ingestión de ooquistes que se encuentran diseminados en los sitios donde los gatos defecan o en alimentos contaminados.

* Emérito del Departamento de Ginecología y Obstetricia del Hospital San Juan de Dios. Cúcuta.

** Bacterióloga de la Universidad Javeriana. Microbiología Médica, Universidad de Los Andes.

*** Bacterióloga de la Universidad Javeriana.
3. Por ingestión de quistes contenidos en carnes que se consumen crudas o insuficientemente cocidas.

4. Por transfusión sanguínea cuando el donante tiene una toxoplasmosis evolutiva.

\section{Formación de anticuerpos}

Si la embarazada tiene un sistema inmunológico intacto, dos a tres semanas después de iniciada la infección se le pueden detectar mecanismos inmunitarios específicos: Anticuerpos Antitoxoplasma IgM e IgG. Los que primero aparecen son los IgM.

Los anticuerpos IgG ascienden de manera gradual para estabilizarse en 6 a 8 semanas a niveles iguales o superiores a 1: 1024, pudiendo alcanzar cifras hasta de 1: 16.384 o más; estos títulos permanecen estables durante 6 meses aproximadamente para descender luego sin llegar a desaparecer: durante toda la vida se encontrarán títulos aproximados a 1: 32 .

Los anticuerpos IgM ascienden con mayor rapidez que los IgG pero sus títulos permanecen bajos, casi nunca superiores a 1: 64. Persisten a estos niveles 4 meses en promedio; inician luego el descenso y desaparecen totalmente entre 4 y 12 meses $(3,9)$. Los anticuerpos específicos clase IgM se forman durante la infección aguda tanto en la madre como en el feto, pero un feto infectado puede no formar anticuerpos IgM in útero.

La presencia de anticuerpos IgA puede ser también un buen indicador de una infección reciente (9). 


\section{Evolución del embarazo}

La gestante puede transmitir la infección toxoplásmica a su producto por vía transplacentaria dando ocasión a la toxoplasmosis congénita.

El embarazo puede terminar en aborto, en parto antes de tiempo, en parto a término, con recién nacido vivo infectado o sano o con mortinato, según la agresividad del toxoplasma y el estado inmunitario de la gestante.

\section{Toxoplasmosis congénita}

De manera global solamente 0.25 a 5 por mil mujeres embarazadas se encuentran en alto riesgo de dar un hijo con Toxoplasmosis Congénita. No toda gestante que se infecta enferma al producto.

El $50 \%$ de los fetos no se infecta porque la inmunidad materna es suficiente para prevenir la infección fetal siempre que existan títulos estables de anticuerpos, así sean bajos. El riesgo de una infección fetal es mayor cuando la embarazada carece de anticuerpos (4).

El $50 \%$ restantes se encuentran infectados al nacer, de los cuales $83 \%$ presentan títulos positivos de Anticuerpos IgG pero sin manifestaciones clínicas, aun cuando algunos desarrollan más tarde lesiones como ceguera uni o bilateral, alteraciones neurológicas severas, retardo mental, dificultad para el aprendizaje. El 17\% restante nace con manifestaciones clínicas $(46 \%$ con daños leves, $28 \%$ con daños graves como coriorretinitis, calcificaciones difusas intracraneanas e hidrocefalia, y $26 \%$ son mortinatos).

\section{Examen de la embarazada}

A toda gestante a quien no se le haya hecho con anterioridad examen serológico para detectar toxoplasmosis o que al practicarlo la respuesta haya sido negativa, se le debe ordenar en la primera consulta prenatal una titulación de anticuerpos antitoxoplasma IgG.

Si el examen solicitado da respuesta negativa, No Reactivo, como sucede en el $15 \%$ de las gestantes, quiere decir que la paciente no ha adquirido infección toxoplásmica. Se debe repetir entonces el examen serológico cada tres meses durante el tiempo que dure el embarazo ante la posibilidad de una seroconversión.
Cuando la investigación de anticuerpos antitoxoplasma IgG es reactiva a cualquier titulación, es preciso diferenciar entre una infección activa, reciente, y una infección latente, anterior. Para ello se pueden seguir dos conductas:

1. Hacer tres semanas más tarde una nueva titulación de IgG.

\section{Investigar de inmediato anticuerpos IgM.}

Si los títulos IgG en el segundo examen aparecen superiores en tres o más diluciones, la infección es reciente; si permanecen estables, la infección es latente o sea anterior al embarazo en curso. Si el IgM es positivo, la infección es reciente.

\section{DIAGNOSTICO}

Hay diferentes métodos para el diagnóstico de la toxoplasmosis, unos de gran utilidad en encuestas epidemiológicas como la intradermo reacción o prueba cutánea de Frenk, o pruebas serológicas de coloración como la de Sabin Feldman, o pruebas de aglutinación.

En la actualidad se emplean métodos inmunológicos que detecten anticuerpos específicos. Las pruebas más usadas son:

1. Inmuno Fluorescencia Indirecta, IFI, que detecta los anticuerpos IgG.

2. La Prueba de REMINGTON que detecta anticuerpos IgM.

3. La prueba de Inmuno Ensayo Enzimático o ELISA, que detecta anticuerpos IgG e IgM. Es una prueba de alta sensibilidad que puede medir niveles bajos de anticuerpos.

4. La prueba de Inmunoperoxidasa es de gran valor. Es una prueba comparable a la IFI con la diferencia que el anticuerpo está marcado con peroxidasa en lugar de fluoresceína. Tiene la ventaja de que la reacción se visualiza en microscopio corriente.

Las pruebas IFI, Remington e Inmunoperoxidasa pueden dar falsos positivos cuando el suero que se investiga contiene Factor Reumatoideo no específico (FR), o anticuerpos antinucleares (ANA). Estos falsos positivos no aparecen cuando se emplea el ELISA. 
El hallazgo del Toxoplasmosis en los tejidos de las pacientes infectadas, aún con manifestaciones clínicas, es muy difícil.

Durante toda la gestación cuando los anticuerpos IgG han aumentado en el segundo examen tres o más diluciones, o el IgM, practicado inmediatamente después de un IgG reactivo, da positivo, la infección ha sido recientemente adquirida, el producto está en riesgo y se impone el tratamiento.

Cuando en el primer trimestre o a principios del segundo los anticuerpos IgG Reactivos permanecen estables en el segundo examen, o los IgM, investigados inmediatamente después de los IgG reactivos, son negativos, la infección toxoplásmica fue adquirida antes del embarazo que cursa y el feto no está en riesgo.

Cuando a finales del segundo trimestre o en el tercer trimestre se encuentran títulos IgG iguales o superiores a 1: 1024 que permanecen estables en el segundo examen, o los anticuerpos IgM, investigados inmediatamente después de los IgG reactivos, son negativos, es imposible saber si la infección se inició antes o después de la concepción. Ante la duda lo racional es empezar el tratamiento.

Cuando en el primer examen los títulos IgG son muy elevados, v.g. 1: 4096 o más, no cabe la menor duda, la infección es reciente y se debe tratar.

Sucede que títulos elevados de IgG pueden bloquear la respuesta de los IgM; por ello se pueden encontrar títulos altos de IgG con IgM negativos. En estos casos es preciso investigar a las tres semanas los anticuerpos IgG para observar si los títulos ascienden o permanecen estables. El ELISA, con su alto nivel de sensibilidad, evita las interferencias causadas por los anticuerpos IgG y detecta siempre los anticuerpos IgM (1).

\section{INTERPRETACION DE LOS TITULOS DE IgG}

\section{Títulos No Reactivos}

Aproximadamente el $15 \%$ de las gestantes no presentan anticuerpos IgG: no han tenido infección toxoplásmica. Se debe investigar IgG cada trimestre durante el embarazo ante la posibilidad de una seroconversión.

\section{Títulos de 1: 16 a 1: 128}

Se encuentran en el $30 \%$ de las embarazadas. Indican que la gestante ha sufrido la infección toxoplásmica y presenta o una infección que se inicia con títulos en ascenso o hay una infección muy anterior que presenta ya anticuerpos permanentes.

\section{Títulos de 1: 256 a 1: 512}

Se observan en el $23 \%$ de las gestantes. Se interpretan como una infección que está en evolución o una toxoplasmosis de iniciación no muy anterior cuyos títulos están en descenso.

\section{Títulos de 1: 1024 o más}

Sugieren una toxoplasmosis evolutiva. Pero en éste como en los casos anteriores un solo examen no hace diagnóstico: es indispensable una segunda titulación tres semanas después u ordenar un Remington de inmediato.

Cuando se practica el ELISA la equivalencia entre los títulos de anticuerpos IgG en relación con los investigados por el IFI, es la siguiente:

\begin{tabular}{|lrll|}
\hline \multicolumn{2}{|c}{ Elisa } & \multicolumn{1}{c|}{ IFI } \\
\hline Menos de & 30 & U.I. & No reactiva \\
& 30 a 80 & U.I. & $1: 16$ a $1: 128$ \\
& 100 a 200 & U.I. & $1: 256$ a $1: 512$ \\
Más de & 250 & U.I. & $1: \quad 1024$ \\
& 300 & U.I. & Mayor de $1: 1024$ \\
\hline
\end{tabular}

\section{TRATAMIENTO}

La mujer embarazada se trata sólo cuando se demuestre que la infección se ha iniciado en el embarazo actual. El tratamiento se instituye como profilaxis para proteger al feto de la Toxoplasmosis Congénita con drogas que no le produzcan efectos teratógenos. Otra indicación, no obstétrica, es cuando la embarazada con serología positiva a cualquier título inicia una retinocoroiditis (8).

Durante el embarazo hay tres drogas de elección: Spiramicina, Clindamicina y Josamycin.

La SPIRAMICINA se concentra en la placenta pero no pasa al feto; se administran $500 \mathrm{mg}$. vía 
oral cada 6 horas durante tres semanas y se puede repetir a intervalos de 15 días. Reduce la transmisión al feto pero no la severidad de la infección trasmitida (1), (3).

La CLINDAMICINA pasa al feto y parece ser inocua a éste; es muy efectiva en toxoplasmosis experimental y de gran utilidad en la infección fetal. Se administra vía oral a la dosis de $300 \mathrm{mg}$. cada 6 horas durante tres semanas y se puede repetir a intervalos de 15 días.

La JOSAMYCINA propionato se concentra a altas dosis en la placenta; actúa a nivel de los ribosomas del toxoplasma e inhibe la síntesis proteica; produce importantes anomalías interkinéticas que le restan capacidad vital.

Se administra por vía oral a la dosis de $1 \mathrm{~g}$ cada 8 horas por diez días y se repite una segunda y una tercera vez con intervalo de 10 días $(2,11)$.

Cuando la gestante adquiere la infección toxoplásmica a fines del segundo o en el tercer trimestre y se encuentran niveles muy altos en la titulación de los anticuerpos desde el primer examen, se debe pensar en la posibilidad de iniciar el tratamiento clásico a base de Pirimetamina más Sulfadiazina o Sulfadoxina, agregando ácido folínico o levadura de cerveza que contrarrestan los efectos tóxicos de las sulfas sin interferir en su efectividad quimioterapéutica. Estas sulfas se disuelven en cantidad suficiente en el líquido intracelular donde se encuentra el toxoplasma, se potencializan y bloquean las dos enzimas que intervienen en la biosíntesis del ácido fólico en el parásito. Se dan a dosis altas en los tres primeros días para que se absorban y se acumulen lentamente en los tejidos (10), (13).

Hay un producto, el FALCIDAR, que contiene en cada comprimido $25 \mathrm{mg}$ de Pirimetamina y $500 \mathrm{mg}$ de Sulfadoxina. Se administra un comprimido cada 8 horas por cuatro días y luego uno cada cuarto día por cuatro a seis semanas.
Las drogas empleadas producen supresión de la enfermedad activa, pero no la erradicación de los organismos enquistados (7).

Todos los recién nacidos de madres tratadas se deben controlar serológicamente para determinar la necesidad de continuar el tratamiento.

\section{PROFILAXIS}

La profilaxis en la mujer embarazada tiene como mira prevenir la toxoplasmosis congénita. Por ello cuando una gestante no presenta títulos de anticuerpos antitoxoplasma debe evitar a toda costa contraer la infección toxoplásmica observando las siguientes normas:

1. Abstenerse de entrar en contacto directo con gatos o con cualquier animal doméstico, mamífero o ave.

2. No manipular la tierra ni las carnes crudas sin el empleo de guantes.

3. Lavarse las manos con jabón antes de cada comida.

4. No consumir carnes crudas o semicrudas.

5. Si hay gato en la casa enseñarlo a defecar en un cajón con tierra y eliminar las heces por cremación.

6. Alimentar los gatos con enlatados.

El uso de anticuerpos monoclonales para aislar antígenos capaces de inducir una resistencia contra el Toxoplasma Gondii hace surgir la posibilidad de que nos estemos acercando al momento en que sea posible la VACUNA contra este organismo (1).

\section{BIBLIOGRAFIA}

1. ARAUJO, F.G. Avances recientes en el diagnóstico e inmunología de la toxoplasmosis. En: Simposio Interamericano de Toxoplasmosis. Memorias. Ed. Guadalupe Ltda. Bogotá, 119, 1984.
2. CAMARASA, A.M.; MONFORT, A.R. Josamycin propionate for the treatment of toxoplasmosis in women at gestational age. 13th International Congress of Chemotherapy. Viena 28th August to 2nd September, 1983. 
3. DAFFOS, F.; FORESTIER, F.; CAPELLA-PAVLOVSKY, $M$. et al. Prenatal management of 746 pregnancies at risk for congenital toxoplasmosis. E. Eng. J. Med. 318: 271, 1988.

4. DESMONTS, G. Toxoplasmose acquise de la femme enceinte. Lyon Medical. 248, 115, 1982.

5. DUARTE-CONTRERAS, A. Criterios para el diagnóstico y el tratamiento de la toxoplasmosis. Rev. Colomb. Obstet. Ginecol. 35: 205, 1984.

6. DUARTE-CONTRERAS, A.; DUARTE, B.C.I.; DUARTE, B.G. de R. Toxoplasmosis y embarazo. Rev. Colomb. Obstet. Ginecol. 38: 209, 1987.

7. FELDMAN, H.A. Toxoplasmosis. En: HARRISON, T.R. Medicina Interna. Ed. La Prensa Médica Mexicana. 1207, 1965.

8. FRENKEL, J.K. El tratamiento de la toxoplasmosis. En: Simposio Interamericano de Toxoplasmosis. Memorias. Ed. Guadalupe Ltda. Bogotá, 95, 1984.
9. KAGAN, I.G. Diagnóstico serológico de Toxoplasmosis. En: Simposio Interamericano de Toxoplasmosis. Memorias. Ed. Guadalupe Ltda. Bogotá, 112. 1984.

10. KAUFMAN, H.E.; CALDWELL, L.A. Pharmacologycal Studies of pyrimethamine (Daraprin) in man. Arch. Ophthal. 61: $885,1959$.

11. MONZONIS, LM.; PERALES, M.A. Josamycin on Toxoplasmosis treatment: results. 13th International Congress of Chemotherapy. Viena 28th August to 2nd September, 1983.

12. NAOT, T. et al. Duration of IgG antibodies to Toxoplasma Gondii after acute acquired toxoplasmosis. Infect. Dis. 145: 770, 1982.

13. SANCHEZ, T.F. Toxoplasmosis y embarazo. En: Alto Riesgo Obstétrico. Ed. Universidad Nacional. Bogotá. 112, 1988.

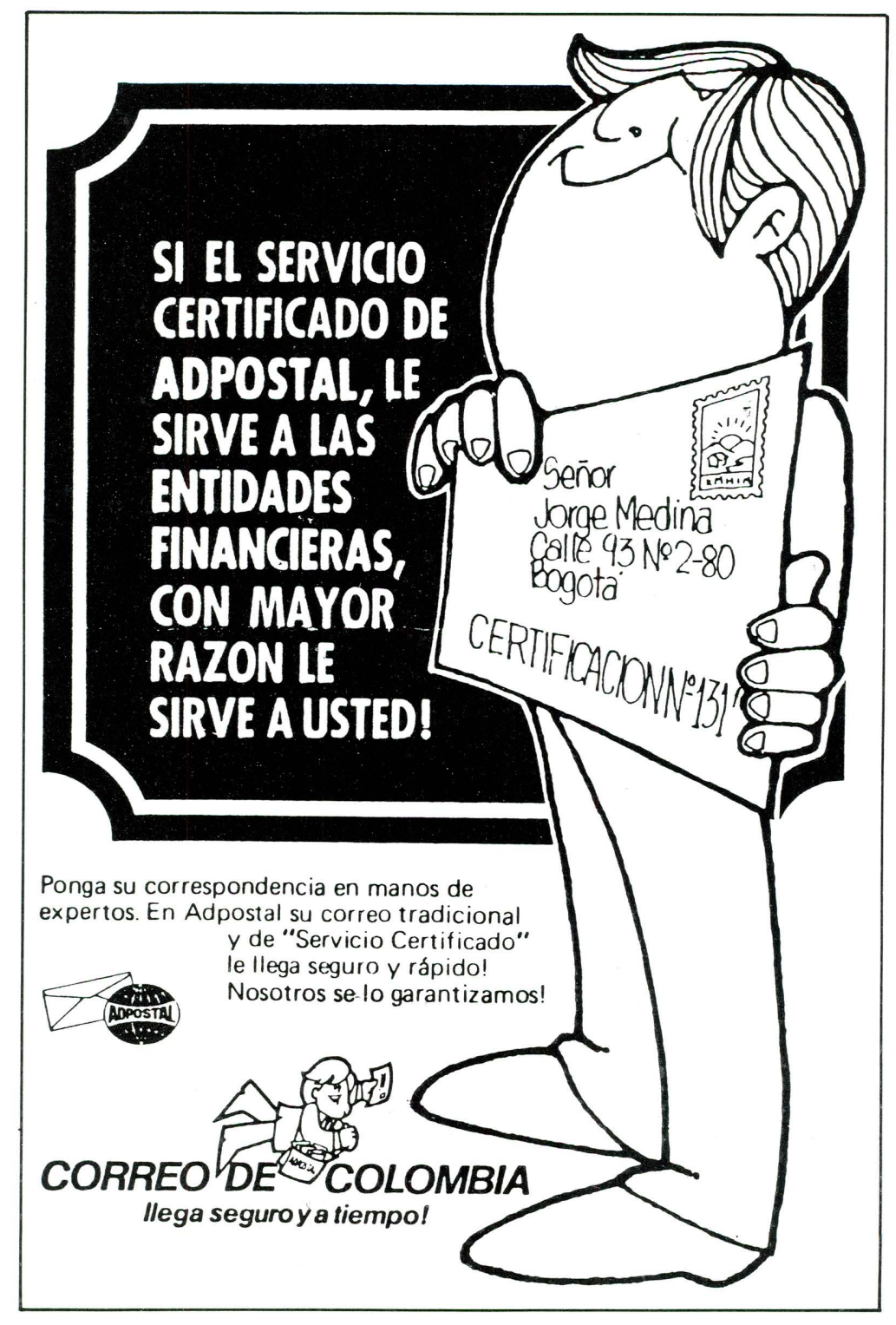

